\title{
Novel Styrylquinolinium Dye Thin Films Deposited by Pulsed Laser Deposition for Nonlinear Optical Applications
}

\author{
H. El Ouazzani, ${ }^{\dagger, \perp}$ Sylvie Dabos-Seignon, ${ }^{\dagger}$ D. Gindre, ${ }^{\dagger}$ K. Iliopoulos, ${ }^{\dagger}$ M. Todorova, ${ }^{\dagger}$ R. Bakalska, ${ }^{\ddagger}$ \\ P. Penchev, ${ }^{\ddagger}$ S. Sotirov, ${ }^{\S}$ Ts. Kolev, ${ }^{\ddagger}$ V. Serbezov, $\|$ A. Arbaoui, ${ }^{\perp}$ M. Bakasse, ${ }^{\perp}$ and B. Sahraoui ${ }^{*}{ }^{\dagger}$ \\ ${ }^{\dagger}$ LUNAM Université, Université d'Angers, CNRS UMR 6200, Laboratoire MOLTECH-Anjou, 2 bd Lavoisier, 49045 ANGERS cedex, \\ France \\ ${ }^{\ddagger}$ Faculty of Chemistry, Plovdiv University “P. Hilendarski”, 24, Tzar Assen Str., 4000 Plovdiv, Bulgaria \\ ${ }^{\S}$ Faculty of Physics, Plovdiv University "P. Hilendarski”, 24, Tzar Assen Str., 4000 Plovdiv, Bulgaria \\ "Vascotec GmbH, Gewerbepark Keplerstr. 10/12, Gera, Germany \\ ${ }^{\perp}$ Laboratory of organic chemistry, bio-organics and environment, Faculty of Science, University Chouaib Doukkali, 24000, El Jadida, \\ Morocco
}

ABSTRACT: The nonlinear optical (NLO) properties of novel styrylquinolinium dye thin films for photonic applications have been studied by the Z-scan, second harmonic generation (SHG), and third harmonic generation (THG) techniques, providing both the second- and third-order nonlinear optical parameters. The styrylquinolinium dye $(E)$ 1-ethyl-4-(2-(4-hydroxynaphthalen-1-yl)vinyl)quinolinium bromide was synthesized by the Knoevenagel condensation, and its structure and physicochemical properties were determined by ${ }^{1} \mathrm{H}$ NMR, ${ }^{13} \mathrm{C}$ NMR, FTIR, UV-vis spectros-
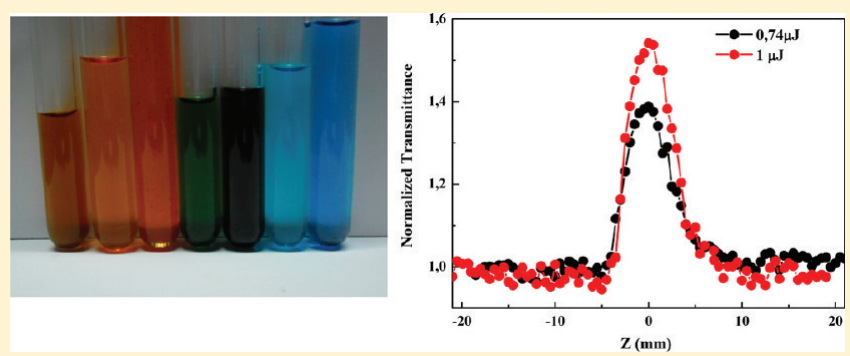
copy, and elemental analysis. Functional thin films were deposited by pulsed laser deposition (PLD) using UV TEA $\mathrm{N}_{2}$ laser onto glass substrates and $\mathrm{KCl}, \mathrm{NaCl}$ monocrystals at room temperature and vacuum at $10^{-3} \mathrm{mbar} / 0.1 \mathrm{~Pa}$. Further characterization of the films and target from native material by FTIR spectroscopy revealed that there was no difference between the deposited films and the initial material. Atomic force microscopy (AFM) and scanning electron microscopy (SEM) measurements have been also performed in order to provide information about the morphology and topology of the thin films.

\section{INTRODUCTION}

Organic nonlinear optical (NLO) materials vary from polymers to molecular crystals, liquid crystals, organometallic compounds, and new hybrid organic/inorganic nanocomposites. Bulk materials or thin film structures with high nonlinearity can be easily used for NLO devices and applications like optical switches, modulators, devices for frequency mixing processes, optical sensors, integrated optical circuits for telecommunications, and optical computing. ${ }^{1}$

The most common design of molecules with large first and second molecular hyperpolarizabilities values comprises of strong electron donors and acceptors connected by a $\pi$ conjugated system (donor- $\pi$-bonding sequence-acceptor or "push-pull" chromophores). ${ }^{2-5}$ The vast majority of pushpull chromophores are organic molecules, with donor $-\pi$ bonding sequence-acceptor, and these three keys components can be varied very widely. Organic compounds with highly polarizable $\pi$-electron systems have attracted much interest in view of their large optical nonlinearity, e.g., the molecular second-order polarizability $\beta$ of hemicyanine dyes is in the range of $10^{-28}-10^{-27}$ esu (or $3.7 \times 10^{-49}-3.7 \times 10^{-48} \mathrm{C} \mathrm{m}^{-3}$ $\mathrm{V}^{-2}$ in the SI unit system), which is at least 2 orders of magnitude greater than most inorganic materials in practical use. $^{6}$

The basic idea of the design of the new styrylquinolinium dye (E)-1-ethyl-4-(2-(4-hydroxynaphthalen-1-yl)vinyl)quinolinium bromide system is to enlarge the $\pi$-conjugated system in the $y$ direction while in the literature systems are described, where $\pi$ conjugation enlargement has been achieved in the $x$-direction by using quinolinium and naphthalene moieties, thus increasing the molecular hyperpolarizabilities.

The crystallization ability of the compound and the mechanical properties are improved with respect to styryl dyes with benzene and pyridine rings. The larger conjugated $\pi$ system is a prerequisite for enhanced nonlinear optical response. It is leading to batochromic shift of $\lambda_{\max }$ and to larger solvatochromism. The melting point of the material is higher $\left(285-287{ }^{\circ} \mathrm{C}\right.$ ) than the known merocyanine dyes, which is a further advantage for the manufacturing of devices. For this dye, high damage threshold can be expected. ${ }^{7-9}$

Received: December 8, 2011

Revised: February 24, 2012

Published: February 29, 2012 
Thin films of complex organic materials have been processed by means of conventional nonvacuum techniques, such as dipcoating, spin-coating, spray coating, electrochemical deposition, Langmuir-Blodgett method, and vacuum methods such as electron beam evaporation, thermally assisted vacuum evaporation, and organic molecular beam epitaxy. ${ }^{10,11}$ The pulsed laser deposition (PLD) technique is well-known for deposition of complex inorganic thin films. Recently developed laser-based methods, such as matrix-assisted pulsed laser evaporation (MAPLE) using high-power UV excimer lasers and resonant infrared PLD (RIR PLD) using free electron laser (FEL), have been found to be very appropriate for deposition of complex organic materials, including polymers, biopolymers, proteins, and living cells. ${ }^{12}$ The intrinsic disadvantages of these techniques are the necessity to use frozen solutions of the organic compounds as MAPLE target and complex/expensive FEL as ablating source for RIR PLD.

The aim of this work is first to employ the conventional PLD technique using a relatively compact and cheap UV TEA $\mathrm{N}_{2}$ laser to produce high quality and functional thin films consisting of novel NLO organic styrylquinolinium materials onto various substrates. The organic styrylquinolinium materials were analyzed by ${ }^{1} \mathrm{H}$ NMR, ${ }^{13} \mathrm{C}$ NMR, FTIR, UVvis spectroscopy, and elemental analysis. The deposited organic thin films were studied by FTIR, SEM, and AFM. Then complete characterization of the second- and third-order nonlinear optical response by means of the Z-scan, ${ }^{13}$ SHG, and $\mathrm{THG}^{14-16}$ techniques has been carried out in order to investigate the possibility to utilize these systems in appropriate photonic applications.

\section{EXPERIMENTAL METHODS}

2.1. Synthesis of $(E)$-1-Ethyl-4-(2-(4-hydroxynaphthalen-1-yl)vinyl)quinolinium Bromide. Starting compounds were commercially available and were used as supplied. Lepidine (4-methylquinoline) and ethyl bromide were freshly distillated. The conversion of the starting materials and the progress of the reactions were followed by thin-layer chromatography (TLC). The TLC was performed on plates of silica gel 60 (Merck) with mobile phases: (A) hexane:ethyl acetate $(1: 2)$; (B) petroleum ether:diethyl ether $(2: 1)$; (C) $n$ propanol:formic acid (4:1).

${ }^{1} \mathrm{H}$ and ${ }^{13} \mathrm{C}$ NMR measurements, referenced to TMS, were made at $298 \mathrm{~K}$ with a Bruker DRX-250 spectrometer using 5 $\mathrm{mm}$ tubes. The dye was dissolved in $\mathrm{CD}_{3} \mathrm{OD}$. The chemical shifts were referenced internally in $\delta$-units. $J_{\mathrm{HH}}$ coupling constants were measured in $\mathrm{Hz}$. Fourier transform infrared spectra were recorded on VERTEX 70 FTIR spectrometer (Bruker Optics). The UV-vis spectra (1 cm quartz cell) were measured on Perkin-Elmer LAMBDA 9 UV-vis/NIR spectrometer.

Preparation of Lepidinium Ethyl Bromide. Ethyl bromide $(1.5700 \mathrm{~g}, 14.45 \mathrm{mmol})$ was dropped into the solution of lepidine $(2.1600 \mathrm{~g}, 14.45 \mathrm{mmol})$ in $6 \mathrm{~mL}$ of dry toluene. The reaction mixture was boiling for $20 \mathrm{~h}$, filtered off, and washed with toluene. Crystallization from absolute ethanol resulted to white crystals of lepidinium ethyl bromide. The resulting material was dried under vacuum and kept over $\mathrm{P}_{2} \mathrm{O}_{5}$. Yield: $59 \%$. The TLC was performed with mobile phase $\mathrm{A} / \mathrm{I}_{2}$ vapor.

Preparation of Dye. A mixture of the lepidinium ethyl bromide $(0.1990 \mathrm{~g}, 0.79 \mathrm{mmol})$, 4-hydroxynaphthaldehyde $(0.1360 \mathrm{~g}, 0.79 \mathrm{mmol})$, piperidine $(0.0067 \mathrm{~g}, 0.079 \mathrm{mmol})$, and glacial acetic acid $(0.0095 \mathrm{~g}, 0.158 \mathrm{mmol})$ in dry benzene (20
$\mathrm{mL}$ ) was heated under reflux, equipped with a Dean-Stark apparatus, and drying tube. After boiling for $6 \mathrm{~h}$ the dark red crystals were filtered off, washed with benzene, and dried. The TLC was performed with mobile phase $\mathrm{B}$ and $\mathrm{C} / \mathrm{I}_{2}$ vapor and UV light. The dye was crystallized from methanol. The obtained compound was dark red crystals with high mp 285-7 ${ }^{\circ}$ C. Yield: $97 \%$.

Elemental analysis of $\mathrm{C}_{23} \mathrm{H}_{20} \mathrm{BrNO}$; calcd $\mathrm{C} 67.99 \%, \mathrm{H}$ 4.96\%, N 3.45\%; found C 68.03\%, H 5.00\%, N 3.47\%. ${ }^{1} \mathrm{H}$ NMR $\left(\mathrm{CD}_{3} \mathrm{OD}\right) \delta 8.97(\mathrm{~d}, J=6.6 \mathrm{~Hz}, 1 \mathrm{H}, \mathrm{H}-2), 8.77(\mathrm{dd}, J=8.5$, $3.5,15.6 \mathrm{~Hz}, 2 \mathrm{H}, \mathrm{H}-8, \mathrm{H}-11$ ), 8.35 (d in $\mathrm{t}, J=8.6,6.8 \mathrm{~Hz}, 3 \mathrm{H}$, H-3, H-5, H-5'), 8.22 (dm, $J=8.4,2.8 \mathrm{~Hz}, 1 \mathrm{H}, \mathrm{H}-7$ ), 8.18 (d, $J$ $\left.=3.1 \mathrm{~Hz}, 1 \mathrm{H}, \mathrm{H}-8^{\prime}\right), 8.05(\mathrm{~d}, J=15.6 \mathrm{~Hz}, 1 \mathrm{H}, \mathrm{H}-12), 7.97(\mathrm{t}, J$ $\left.=7.4,7.3 \mathrm{~Hz}, 2 \mathrm{H}, \mathrm{H}-2^{\prime}, \mathrm{H}-7^{\prime}\right), 7.59(\mathrm{dtd}, J=1.4,3.1,1.6,6.8$ $\mathrm{Hz}, 1 \mathrm{H}, \mathrm{H}-6), 7.45$ (t, $\left.J=7.2,7.1 \mathrm{~Hz}, 1 \mathrm{H}, \mathrm{H}-6^{\prime}\right), 6.91$ (d, $J=8.2$ $\left.\mathrm{Hz}, 1 \mathrm{H}, \mathrm{H}-3^{\prime}\right), 4.93$ (q, J=7.3 Hz, $\left.\mathrm{CH}_{2}\right), 1.70(\mathrm{t}, J=7.2,7.3 \mathrm{~Hz}$, $3 \mathrm{H}, \mathrm{CH}_{3}$ ). ${ }^{13} \mathrm{C}$ NMR (CD $\left.\mathrm{OD}\right): 159.03$ (C-4), 155.47 (C-4'), 147.11 (C-2), 141.38 (C-10), 139.47 (C-9'), 136.35 (C-7), 134.67 (C-10'), 130.34 (C-7'), 129.01 (C-1'), 128.95 (C-9), 128.47 (C-8'), 127.76 (C-8), 126.27 (C-11), 126.22 (C-6'), 124.65 (C-3), 124.23 (C-5), 123.77 (C-5'), 119.67 (C-2'), 118.80 (C-12), 117.02 (C-6), 109.78 (C-3'), $53.7\left(\mathrm{CH}_{2}\right), 15.5$ $\left(\mathrm{CH}_{3}\right)$.

2.2. Film Deposition. The experimental setup used for the thin film deposition is typical of the PLD technique and is similar to that described in ref 17. For the needs of the deposition, an EMG 120 Lambda Physik UV TEA $\mathrm{N}_{2}$ laser at $337.1 \mathrm{~nm}$ with $5 \mathrm{~ns}$ pulse duration and repetition rate of $20 \mathrm{~Hz}$ has been used (pulse energy $5 \mathrm{~mJ}$ ). The laser beam has been focused by means of a quartz meniscus short focus $(f=5 \mathrm{~cm})$ lens, while the laser energy density fluence has been varied from $200 \mathrm{~mJ} / \mathrm{cm}^{2}$ to $3.5 \mathrm{~J} / \mathrm{cm}^{2}$. The target holder has been rotated with a speed up to $500 \mathrm{rev} / \mathrm{min}$ by means of a high speed, mini motor stage fixed on a single axis scanner inside a vacuum chamber. Using high speed rotation and scanning of target enabled uniform erosion of the target and deposition of the films on relatively large area, up to $10 \mathrm{~cm}^{2}$ without film's thickness gradient. The distance between the target and the substrate (S) was fixed at $4.5 \mathrm{~cm}$. The single bulk dye target has been prepared with the aid of a hydraulic press (Perkin-Elmer) with $1.2 \mathrm{~cm}$ diameter and $0.3 \mathrm{~cm}$ thickness. All the experiments have been carried out at room temperature and vacuum of 0.1 $\mathrm{Pa}\left(10^{-3} \mathrm{mbar}\right)$, which has been achieved by using two-stage standard rotary pumps. The deposition rate was 1-2 $\AA$ /pulse, which is typical for the PLD technique. The microscope glass substrates ("Ilmglass" $20 \times 20 \times 0.22 \mathrm{~mm}, 316 \mathrm{~L} \mathrm{SS}$ alloy, and Al foil) have been cleaned in an ultrasound bath with pure acetone and ethanol, before the deposition process and dried by means of technical nitrogen. Monocrystals $\mathrm{KBr}$ and $\mathrm{NaCl}$ have been used as substrates for the infrared spectroscopy. The choice of substrates has been done in order to provide compatibility with NLO devices. The NLO properties of the deposited styrylquinolinium dye (E)-1-ethyl-4-(2-(4-hydroxynaphthalen-1-yl)vinyl)quinolinium bromide thin films with thickness $60-120 \mathrm{~nm}$, measured by mechanical profilometer, were investigated on "Ilmglass" substrates.

2.3. Nonlinear Optical Techniques. The target of the NLO measurements was the investigation of both the secondand third-order optical nonlinearities of the styrylquinolinium bromide dye thin films. In this direction the Z-scan, third harmonic generation (THG), and second harmonic generation (SHG) Maker fringes experimental techniques have been employed, using a 30 ps diode pumped passively mode-locked 
$\mathrm{Nd}: \mathrm{YVO}_{4}$ laser with a repetition rate of $10 \mathrm{~Hz}$, while the temporal and spatial profile of the beam was Gaussian. The SHG/THG measurements have been carried out employing the fundamental of the laser (at $1064 \mathrm{~nm}$ ) wavelength, while for the Z-scan investigation the frequency-doubled beam (532 $\mathrm{nm}$ ) has been used. By means of the THG setup the magnitude of the third-order nonlinear susceptibility $\left(\chi^{(3)}\right)$, related only to the electronic contribution has been determined, as this technique is not sensitive to slower mechanisms like molecular orientation, redistribution, thermal effects, etc. Additionally, the "open aperture" Z-scan measurements have been performed in order to separately determine the imaginary part of the thirdorder nonlinear susceptibility $\left(\operatorname{Im} \chi^{(3)}\right)$ and the nonlinear absorption coefficient $(\beta)$, which is related to the nonlinear absorption of the samples. The advantage of the Z-scan measurements is that, apart from the magnitude, they can also provide the sign of the $\operatorname{Im} \chi^{(3)}$, while the latter is directly related with the nonlinear absorption type (saturable absorption (SA) or reverse saturable absorption (RSA)). Moreover, with the SHG technique the effective value of the second-order nonlinear susceptibility $\left(\chi^{(2)}\right)$ has been determined. The combination of all these experimental techniques provided global knowledge of the nonlinearity of the systems.

The details of the experimental techniques, as well as the procedure to analyze the experimental data, will be briefly described here, as extended information can be found in the literature. ${ }^{13-16}$ Concerning the "open aperture" Z-scan technique the total transmittance through the film has been measured as it moved along the transmittance axis of the focused laser beam and around the focal plane of the lens. In this way the size of the laser beam on the film was varying, modifying the incident laser intensity. All the transmitted light has been collected with a large diameter focusing lens and measured with a suitable photomultiplier. The measurements have been performed for several different laser energies in order to verify the linear dependence of the nonlinear optical response upon the energy. Also, measurements were carried out in several areas of the film to prove that the response was homogeneous all over the surface. From the "open aperture" Zscan curves the nonlinear absorption coefficient $(\beta)$ has been determined using the equation

$$
\begin{aligned}
T & =\frac{1}{\sqrt{\pi}\left(\frac{\beta I_{0} L_{\text {eff }}}{1+z^{2} / z_{0}^{2}}\right)} \int_{-\infty}^{+\infty} \\
& \times \ln \left[1+\frac{\beta I_{0} L_{\text {eff }}}{1+z^{2} / z_{0}^{2}} \exp \left(-t^{2}\right)\right] \mathrm{d} t
\end{aligned}
$$

where $T$ is the normalized transmittance, $L_{\text {eff }}=(1-$ $\left.\exp \left(-a_{0} L\right)\right) / a_{0}$ is the effective thickness of the sample, $\alpha_{0}$ is the linear absorption coefficient of the sample at the laser excitation wavelength, $L$ is the sample thickness, and $I_{0}$ is the on-axis irradiance at the focus. From the nonlinear absorption coefficient the imaginary part of the third-order nonlinear susceptibility has been determined using the equation ${ }^{13}$

$$
\operatorname{Im} \chi^{(3)}(\mathrm{esu})=\frac{10^{-7} c^{2} n_{0}^{2}}{96 \pi^{2} \omega} \beta\left(\mathrm{cm} \mathrm{W}^{-1}\right)
$$

where $c$ is the speed of light in $\mathrm{cm} \mathrm{s}^{-1}, n_{0}$ is the linear refractive index, and $\omega$ is the fundamental frequency in cycles $\mathrm{s}^{-1}$.
Concerning the SHG and THG Maker fringes setups, the measurements have been carried out in transmission configuration. By means of a $\lambda / 2$ and a polarizer the intensity and the polarization of the fundamental beam on the sample have been adjusted, while two photodiodes have been utilized, for the triggering of the electronic part of the setup and recording the laser intensity on the sample. The second/third harmonic generated photons have been detected by a suitable photomultiplier, and experimental curves of the SHG/THG have been recorded as a function of the angle of incidence, which has been precisely controlled, by a motorized rotation stage. Before the detection, the beam was passing through a KG3 filter, which cut out the fundamental beam, and interference filters, at 532 $\mathrm{nm}$ for SHG and $355 \mathrm{~nm}$ for THG, to preserve the SHG/THG signals, respectively.

For the analysis of the SHG experimental findings many appropriate models can be found in the literature. ${ }^{14,15,18,19}$ Here, the model described in ref 18 has been used as it offers the possibility to take into account the linear absorption, which has been important for the samples studied and around the SHG wavelength $(532 \mathrm{~nm})$. A $0.5 \mathrm{~mm}$ thick Y-cut quartz slab has been used as reference material for these measurements with $\chi^{(2)}=8.07 \times 10^{-12}$ esu $(1 \mathrm{pm} / \mathrm{V})$ and coherence length $20.5 \mu \mathrm{m}$.

For the analysis of the third harmonic generation data the model of Kubodera and Kobayashi ${ }^{20}$ has been used, which provides the third-order nonlinear susceptibility by comparing the emanating signal from the film directly with that of a reference material which has known nonlinear optical response. In the case of significant linear absorption this model becomes

$$
\chi^{(3)}=\frac{2}{\pi} \chi_{s}^{(3)} l_{c}^{S} \frac{\alpha / 2}{1-\exp (-\alpha l / 2)} \sqrt{\frac{I_{3 \omega}}{I_{3 \omega}^{S}}}
$$

In this equation $\chi^{(3)}$ and $\chi_{\mathrm{s}}^{(3)}$ are the third-order nonlinear susceptibilities of the sample and the reference material (silica in this case), respectively, $l_{c}^{\mathrm{S}}$ is the coherence length of fused silica, $l$ is the film thickness, $\alpha$ is the linear absorption coefficient, and $I_{3 \omega}$ and $I_{3 \omega}^{S}$ are the peak intensities of the Maker fringes pattern of the film and the fused silica slab, respectively. The utilized reference $\chi^{(3)}$ value at $1064 \mathrm{~nm}$ for fused silica was $\chi_{\mathrm{s}}^{(3)}=2.0 \times 10^{-22} \mathrm{~m}^{2} \mathrm{~V}^{-2}$. Equation 3 allows the determination of the third-order nonlinear optical response in the case of films, under the prerequisite that the thickness of the film is much lower than the coherence length and also that the signal coming from the substrate can be considered to be negligible, compared to the signal emanating from the film. The latter has been verified in our work by in situ removing a part of the film and measuring the signal from the substrate. During the measurements the films have been positioned with the thin film side facing the detector. The contribution of the substrate has been found to be significant only in the case of the THG measurements and has been in all cases taken into account during the analysis of the experimental data.

\section{RESULTS AND DISCUSSION}

3.1. Synthesis and Spectral Characterization of $(E)-1-$ Ethyl-4-(2-(4-hydroxynaphthalen-1-yl)vinyl)quinolinium Bromide. The novel styrylquinolinium dye was synthesized by alkylation of 4-methylquinoline and consequent Knoevenagel condensation of respective quaternary salt lepidinium ethyl bromide and 4-hydroxynaphthaldehyde in the presence of catalyst piperidine/acetic acid in solvent benzene. The reaction 
scheme for obtaining of compound studied is shown in Scheme 1.

Scheme 1. Reaction Scheme for Obtaining of (E)-1-Ethyl-4(2-(4-hydroxynaphthalen-1-yl)vinyl)quinolinium Bromide<smiles>CC[n+]1ccc(C)c2ccccc21</smiles>

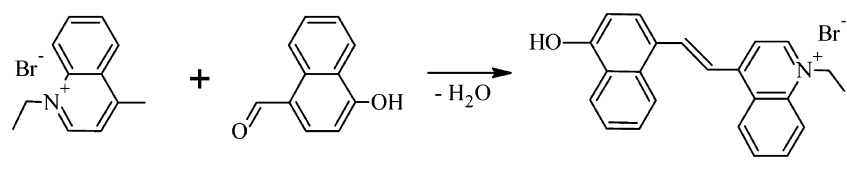

The structure of the newly synthesized styrylquinolinium dye was confirmed by FTIR, ${ }^{1} \mathrm{H}$ NMR, ${ }^{13} \mathrm{C}$ NMR, UV-Vis spectroscopy, and elemental analysis. The obtained dye is partially soluble in nonpolar organic solvents and readily soluble in polar organic solvents giving colored solutions. The color depends on the type of the utilized solvent, as it can be seen in Figure 1. It exhibits in general solute-solvent

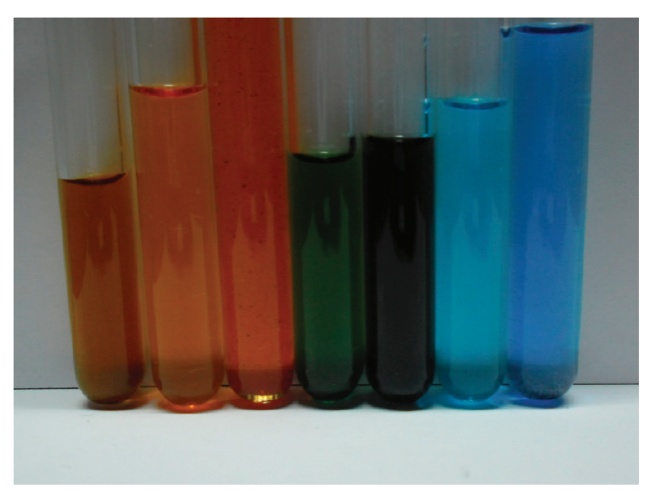

Figure 1. Photography of the solutions of investigated dye in different solvents-from left to right: water, THF, acetonitrile, acetonitrile (higher concentration), acetone, ethanol, and 1,4-dioxane.

interaction depending on the dielectric constant of the solvent used as well as specific solute-solvent interaction. ${ }^{21-26}$ In Figure 2 are given the UV-vis spectra of the dye in solvents water, methanol, ethanol, THF, and glycerol in concentration range $1 \times 10^{-5}-2.5 \times 10^{-5} \mathrm{~g} \mathrm{~mol} / \mathrm{L}$.

The analysis of the electronic absorption spectra clearly indicates the presence of some absorption bands. The more intensive absorption band in the wavelength region 460-493 $\mathrm{nm}$ corresponds to the $\mathrm{S}_{0}-\mathrm{CT}$ (where $\mathrm{S}_{0}$ is the singlet state and $\mathrm{CT}$ is the charge transfer state) transition. That absorption is caused by intramolecular charge transfer from the oxygen atom (hydroxyl group) to the quaternary nitrogen atom in the quinolinium moiety.

The investigated dye is quinolinium salt containing cation and anion, and they are easy to form stable complex with polar solvent in ground state for electrostatic attraction, which may increase the energy difference $(\Delta E)$ of HOMO-LUMO, resulting equilibrium-excited state level shifts to a higher energy in the polar solvent. ${ }^{21}$

The absorbance spectrum recorded in water solution shows the band with $\lambda_{\max }=460 \mathrm{~nm}$. The highest intense band in the $\mathrm{UV}-$ vis spectrum of methanol solution lies at $493 \mathrm{~nm}$ and

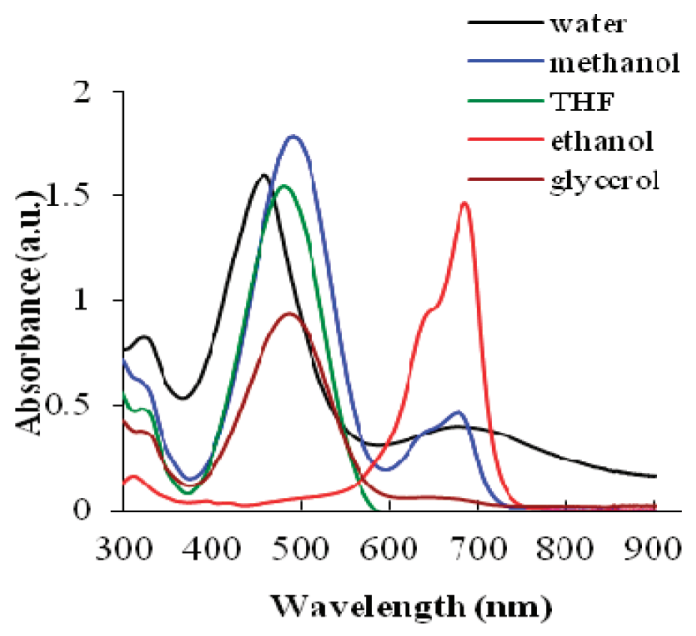

Figure 2. UV-vis spectra of the dye in different solvents: water, methanol, THF, ethanol, and glycerol.

lower intensity bands at 678 and $635 \mathrm{~nm}$ (shoulder), while in ethanol are measured bands at $688 \mathrm{~nm}$ with clearly distinct shoulder $644 \mathrm{~nm}$. That phenomenon could be explained with the differences between dielectric constant of methanol $(\varepsilon=$ 33) and ethanol $(\varepsilon=24.5)$. The solvatochromic effect from water to ethanol is $228 \mathrm{~nm}\left(8094 \mathrm{~cm}^{-1}\right)$. Hence, the experimental results show that the $\lambda_{\max }$ increase with decrease of solvent polarity. The dye studied manifests negative solvatochromism, characteristic for compounds with dipolar electronic ground state structure.

The fluorescence spectrum of the dye in solvent methanol is presented on the Figure 3. The very large Stokes's shift 140.5

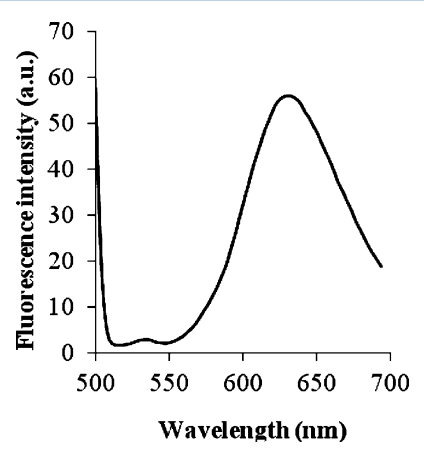

Figure 3. Fluorescence spectrum of the dye in methanol.

$\mathrm{nm}\left(4988 \mathrm{~cm}^{-1}\right)$ was observed in methanol solution. Fluorescing molecules such as the one studied in this work possess the possibility of multiple structures because of the flexibility of the styryl bond, and this should be reflected in their Stokes's shift. Our results are in accordance with an investigation of Jedrzejewska et al. ${ }^{25}$ There are three possible structures of hemicyanine dye (trans, quinoid, and cis). Even though the trans and quinoid structures are mesomeric resonance structures, it should be noted that for two isomers the positive charge is localized on different atoms: on the quinoline nitrogen in trans isomer and on the hydroxyl oxygen in the quinoid form. As a result, solvents of different polarity may interact differently with the two isomers, making possible that either trans or quinoid forms are present in various solvents. 


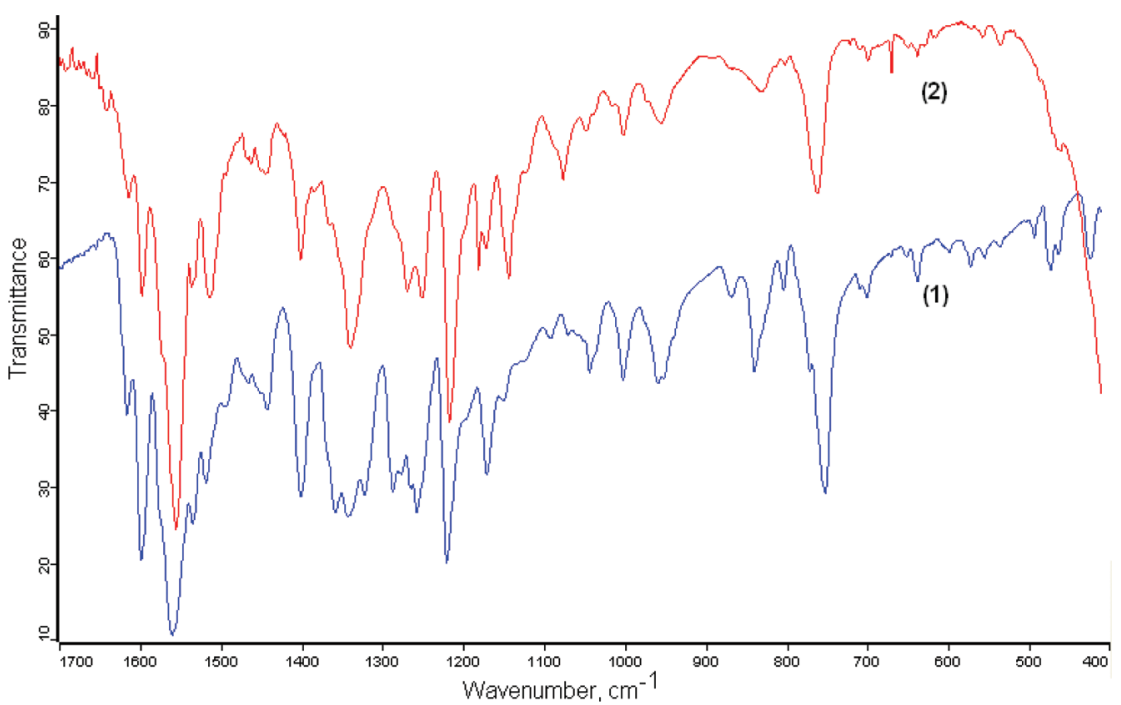

Figure 4. FTIR spectra of (E)-4-(2-hydroxynaphthalen-1-yl)vinyl)-1-ethylquinolinium bromide in the region $1700-400 \mathrm{~cm}^{-1}: \mathrm{curve}^{(1)}$, native material; curve (2), film with thickness $100 \mathrm{~nm}$ onto $\mathrm{NaCl}$ substrate at laser fluence $250 \mathrm{~mJ} / \mathrm{cm}^{2}$.

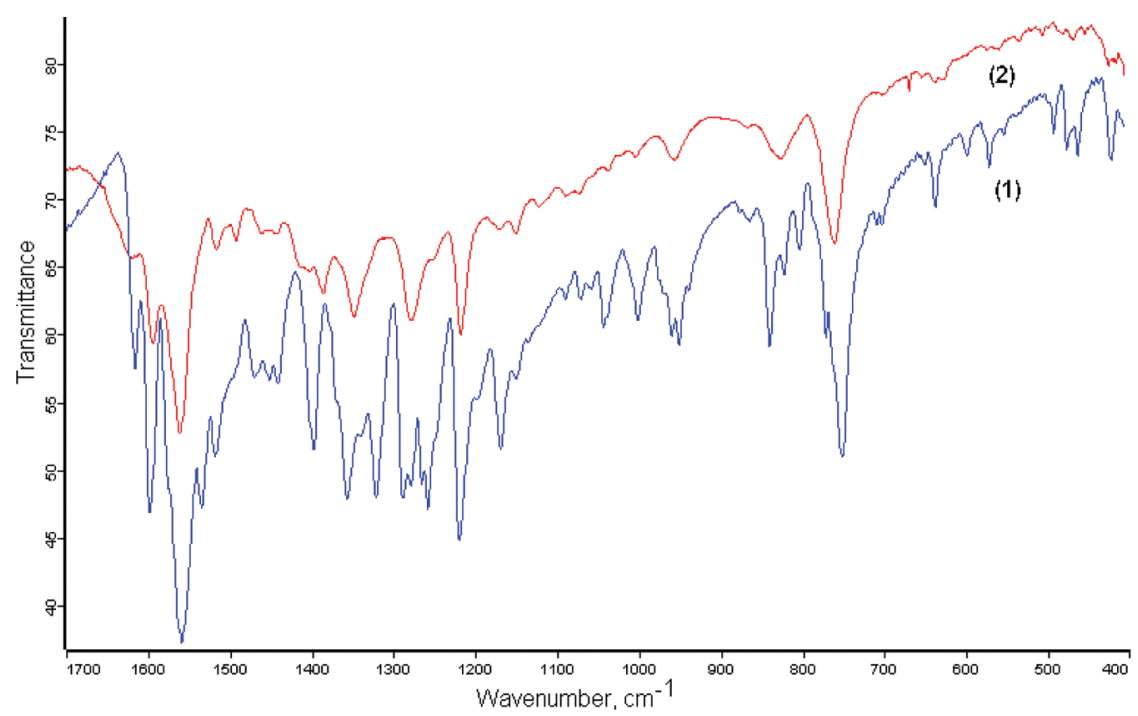

Figure 5. FTIR spectra of (E)-4-(2-hydroxynaphthalen-1-yl)vinyl)-1-ethylquinolinium bromide in the region $1700-400 \mathrm{~cm}^{-1}: \mathrm{curve}(1)$, native material; curve (2), film with thickness $130 \mathrm{~nm}$ onto $\mathrm{KBr}$ substrate at laser fluence $3.5 \mathrm{~J} / \mathrm{cm}^{2}$.

The solvatochromism and the very large Stokes's shift are assigned generally to the change of the molecular dipole moment upon the electronic excitation and the interaction of the dipole moment with the polarizable environment. ${ }^{22}$ This large charge displacement is an indication of a large polarizability. ${ }^{23,24}$ Therefore, the new styrylquinolinium dye can be interesting as a very promising material for NLO applications.

The infrared spectrum of the compound studied is given in Figures 4 and 5, curve (1). It is typical for cationic dyes. The most prominent IR bands at 1558 and $1565 \mathrm{~cm}^{-1}$ correspond to the strongly coupled $\nu_{\mathrm{C}=\mathrm{C}}$ and $8 \mathrm{a}, 8 \mathrm{~b}$ in-plane vibrations of the quinoline fragments in the molecules. These are the characteristic bands that are strongly influenced by the tautomeric effects depending on the solvent polarity. The bands observed within the $3419-3344 \mathrm{~cm}^{-1}$ IR region could be assigned to the stretching vibration of $\mathrm{OH}$ group, in the 4position, which participates in hydrogen bond with $\mathrm{Br}$ anion. The law intense maxima between 3060 and $3010 \mathrm{~cm}^{-1}$ can be attributed to $\mathrm{C}-\mathrm{H}$ stretching vibrations of aromatic rings while the middle intense bands in $2963-2922 \mathrm{~cm}^{-1}$ belong to the $\nu_{\mathrm{C}-\mathrm{H}}$ of the aliphatic chain.

3.2. FTIR Spectral Measurements. The spectral analysis of the materials deposited by means of PLD on various substrates is based on comparison of the spectral bands of the obtained thin films with those of the native material. The comparison between the conventional IR spectrum of the compound studied in $\mathrm{KBr}$ pellet and deposited film on the $\mathrm{NaCl}$ plate manifests practically no considerable differences in both spectra at $250 \mathrm{~mJ} / \mathrm{cm}^{2}$ laser fluence, as shown in Figure 4 . The most prominent band is that at $1558 \mathrm{~cm}^{-1}$ which belongs to the strongly coupled $\nu_{\mathrm{C}=\mathrm{C}}$ and in-plane vibrations of the quinoline fragment in the molecule. This band is the strongest one in the IR spectrum, and its intensity is connected with the charge transfer in the molecule of the $(E)-4$ (2-hydroxynaphthalen-1-yl)vinyl)-1-ethylquinolinium bromide. The higher laser fluence $\left(3.5 \mathrm{~J} / \mathrm{cm}^{2}\right)$ used for the dye deposition in the similar conditions decomposed heavily the target material, and 


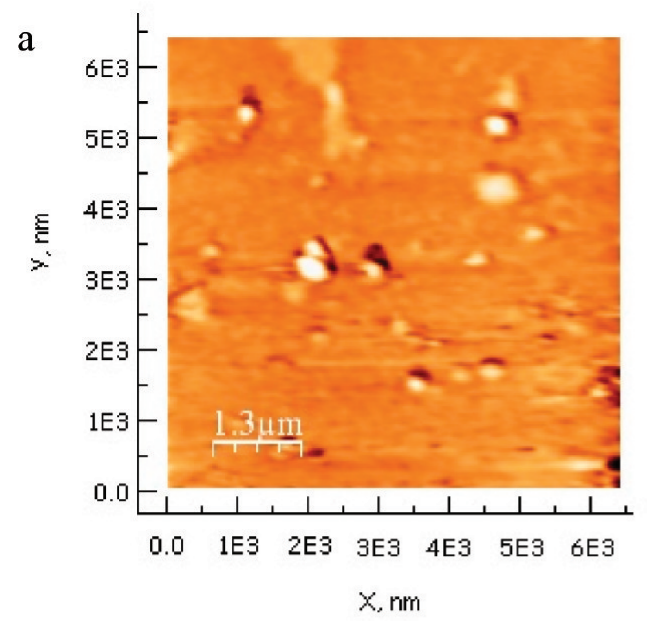

b
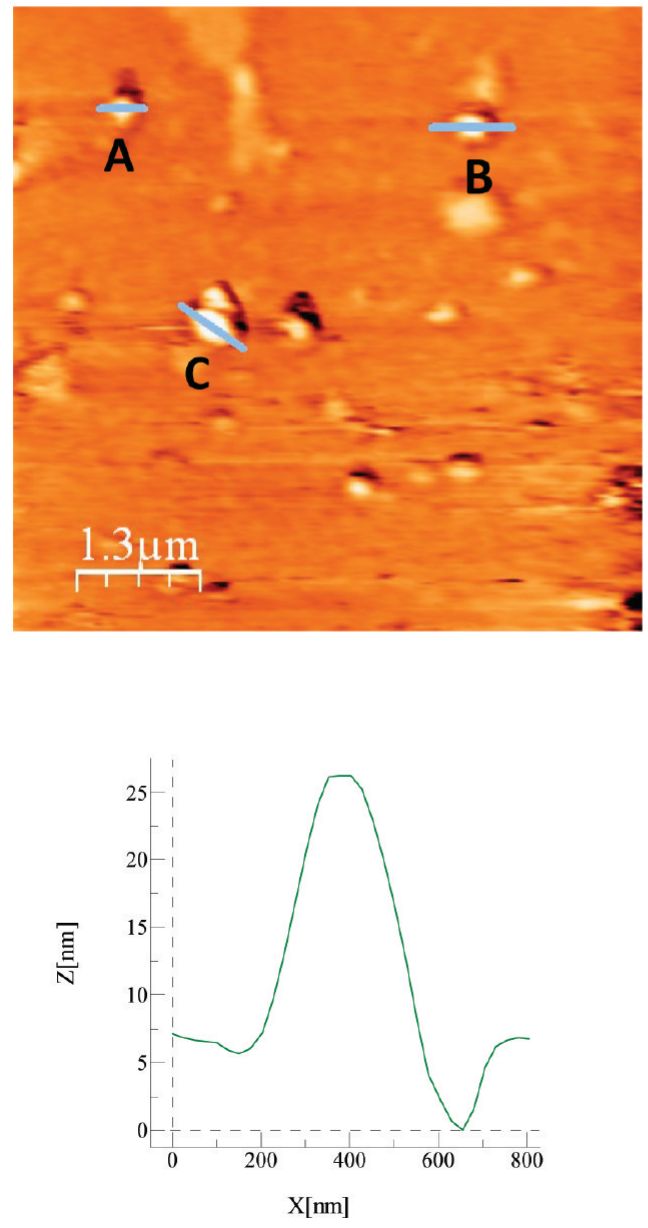

B
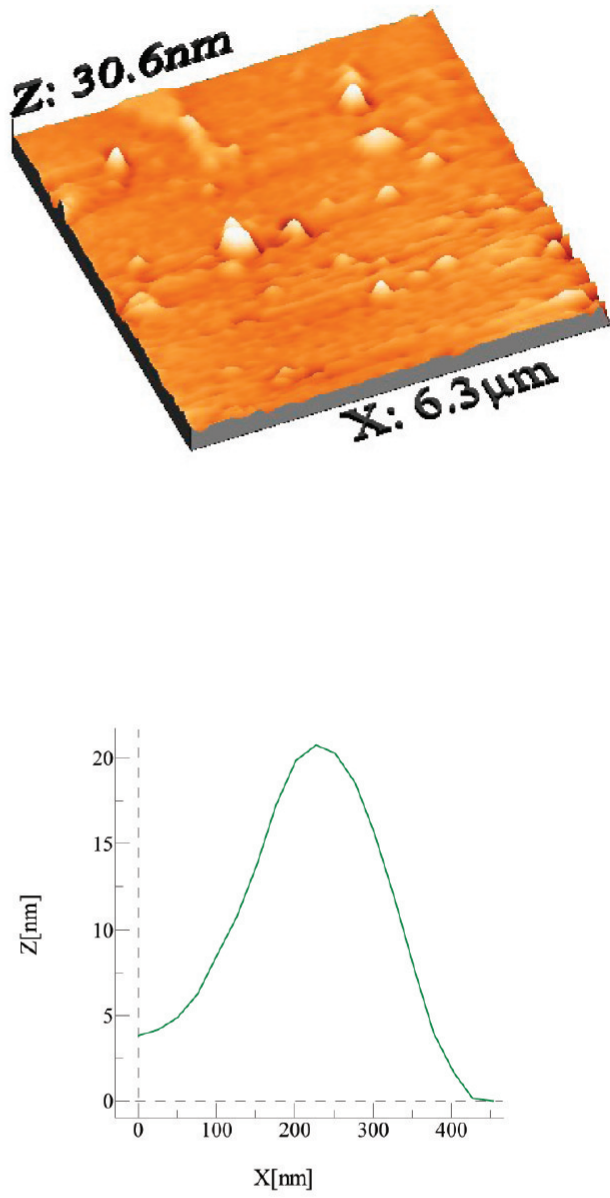

A

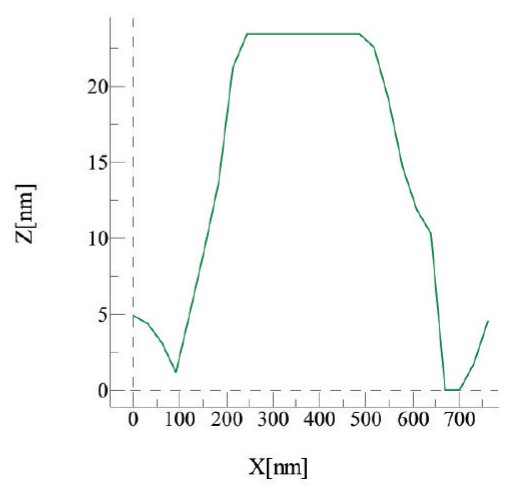

C

Figure 6. (a) 2D and 3D AFM image of the investigated films. (b) Profile at several areas of the AFM image.

the obtained films show different bands compared with the starting compound (Figure 5).The laser fluence and the chemical structure of the dye have been found to be very important parameters for the film degradation, as they were related with the chemical degradation threshold during the deposition process.

3.3. Surface Analysis. Good adhesion of the films on the substrates has been found, while no droplets were observed on the surface of the films, which is a common problem emerging with the PLD method. The films were amorphous and transparent in the visible spectrum.

Atomic force miscroscopy (AFM) measurements were performed using a PicoSpm microscope (Molecular Imaging) in contact mode. Maximum scan size was $6.5 \mu \mathrm{m} \times 6.5 \mu \mathrm{m}$. The obtained raw images were processed using the WSxM program. ${ }^{27}$ Figure 6 shows the planar AFM image after the 
necessary processing. The obtained images show a quite smooth surface, with some fluctuations which can be due to dust deposition related to the manipulation of the sample. The roughness of the samples has been calculated from scans performed on different areas of the samples and found to be about $R=3 \mathrm{~nm}$.

The film morphology has been further examined with highresolution SEM mircroscopy. The SEM representative micrographs of thin films from styrylquinolinium dye with thickness $100 \mathrm{~nm}$ deposited onto glass substrate are shown in Figure 7a,b. The SEM micrographs exhibited very fine texture with
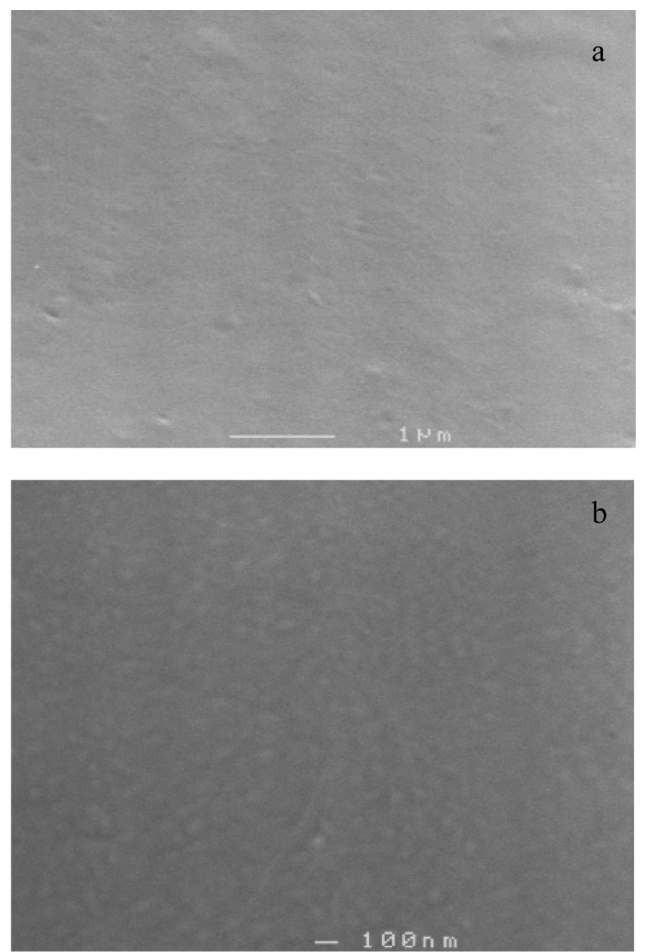

Figure 7. SEM images of the investigated films.

nanoscale dimensions, about $100 \mathrm{~nm}$. The scales can be seen in the figures. From these findings it is obvious that the studied films have been homogeneous without droplets or cracks, verifying the high quality of the surfaces.

3.4. NLO Properties Measurements. For the determination of the nonlinear optical parameters the exact knowledge of the thickness of the investigated films is very important, so it has been separately measured for each film with a profilometer. In all cases it has been found to vary in the range of 60-100 $\mathrm{nm}$. A characteristic absorption spectrum of the films can be seen in Figure 8. It is obvious that there is significant absorbance around $532 \mathrm{~nm}$, which has been the wavelength employed for the Z-scan measurements, as well as the wavelength of the second harmonic generated photons. In both cases the absorbance has been taken into account during the analysis of the experimental data in order to accurately determine the nonlinear optical parameters. The linear absorption coefficient as derived from the absorption spectra at $532 \mathrm{~nm}$ was $\alpha_{0}=7 \times 10^{4} \mathrm{~cm}^{-1}$.

First "open aperture" Z-scan measurements were carried out for the thin films, and several recordings have been acquired for different laser energies. In Figure 9, characteristic traces obtained at 0.74 and $1 \mu \mathrm{J}$ can be seen. A profound

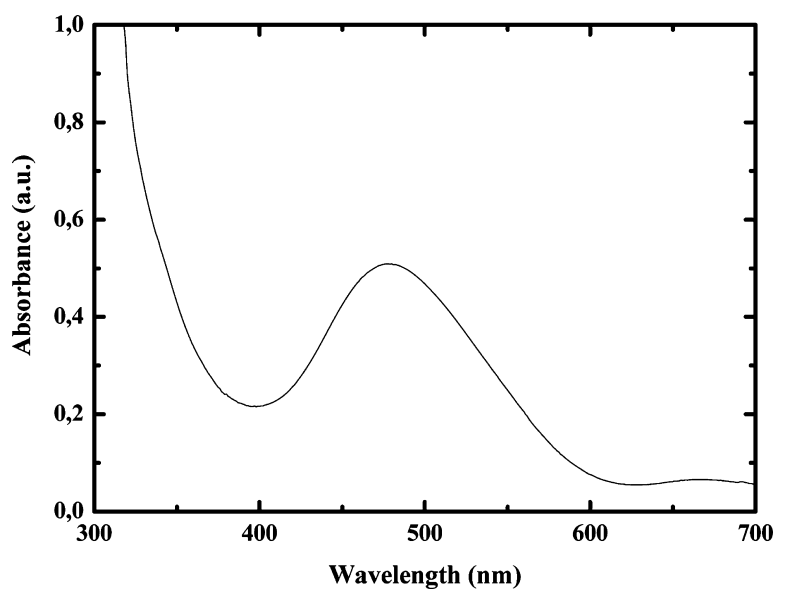

Figure 8. Electronic absorption spectrum of the thin film.

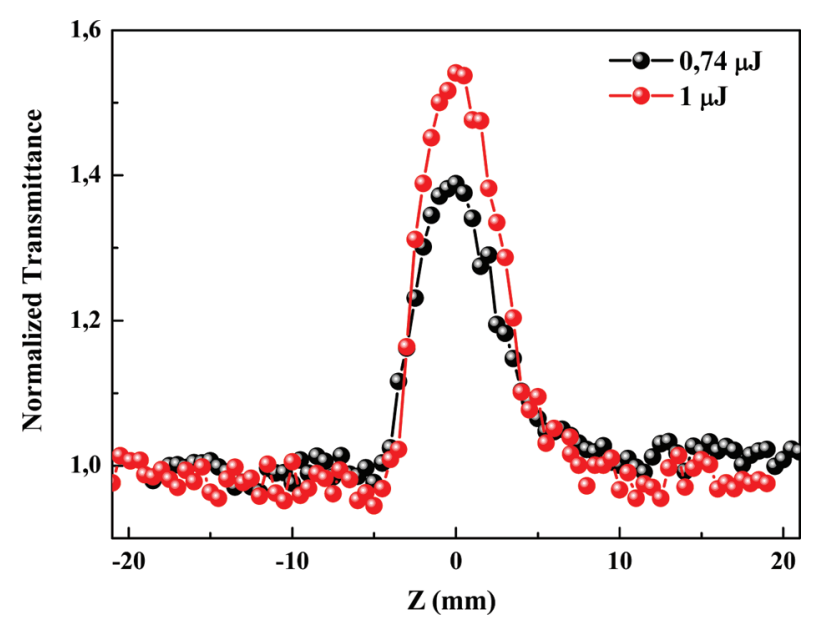

Figure 9. Characteristic "open aperture" Z-scans obtained for the thin films.

transmittance peak can be seen around the focal plane, which is characteristic of saturable absorption (SA)-type behavior of the sample. From a plethora of recordings the $\operatorname{Im} \chi^{(3)}$ has been determined according to the eqs 1 and 2 and has been found to be $\operatorname{Im} \chi^{(3)}=-(2.1 \pm 0.4) \times 10^{-9}$ esu, which corresponds to a nonlinear absorption coefficient of $\beta=-(3.3 \pm 0.7) \times 10^{-7} \mathrm{~m} /$ W. Good dependence of the obtained signals upon the incident laser energy has been found verifying that no saturation of the response was present during the measurements. Measurements carried out in several areas on the film proved that the response was homogeneous and in all cases the obtained data could be fitted perfectly with respect to eq 1 .

Then, SHG Maker fringes measurements have been done for the thin films for $\mathrm{p}$ and $\mathrm{s}$ excitation polarizations. Characteristic Maker fringes experimental curves of the SHG signal, as a function of the angle of incidence of the laser beam with respect to the sample, can be seen in Figure 10, for $s$ and $p$ polarizations, obtained under the same experimental conditions. A big difference of the SHG response between $\mathrm{s}$ and $\mathrm{p}$ polarizations has been found as can be seen in the same figure, while the SHG efficiency for $p$ excitation has been in all cases higher. The contribution of the substrate was negligible with respect to the signal emanating from the film, so the whole signal has been attributed to the film. The effective $\chi^{(2)}$ values have been determined to be 1.40 and $0.95 \mathrm{pm} / \mathrm{V}$ for $\mathrm{p}$ and $\mathrm{s}$ 


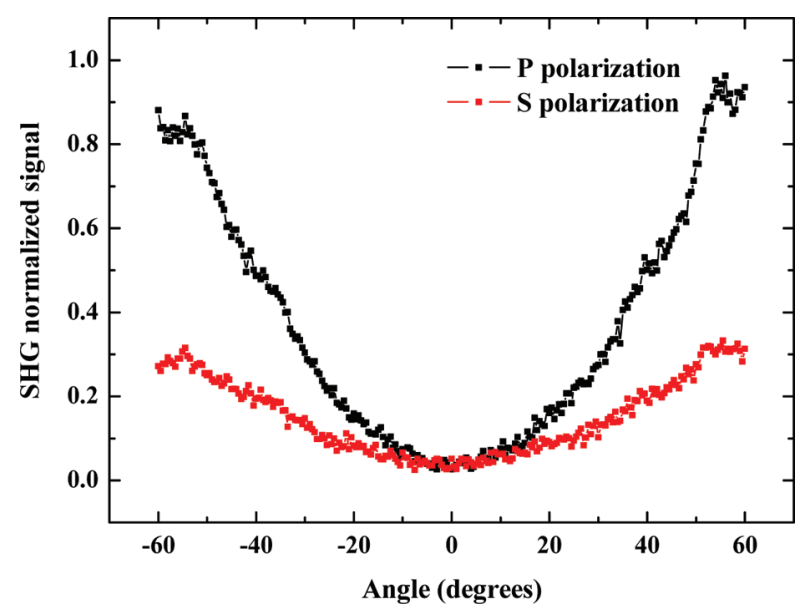

Figure 10. SHG Maker fringes curves for $p$ and $s$ excitation polarizations.

polarization, respectively, so the $\chi^{(2)}$ in the p polarization case is enhanced by about $45 \%$, with respect to the s case.

For the same types of polarization (i.e., $s$ and $p$ cases) THG Maker fringes measurements were carried out. A normalized Maker fringes pattern, showing the oscillatory behavior of the THG response as a function of the angle of incidence, can be seen in Figure 11 in the case of p polarization. The contribution

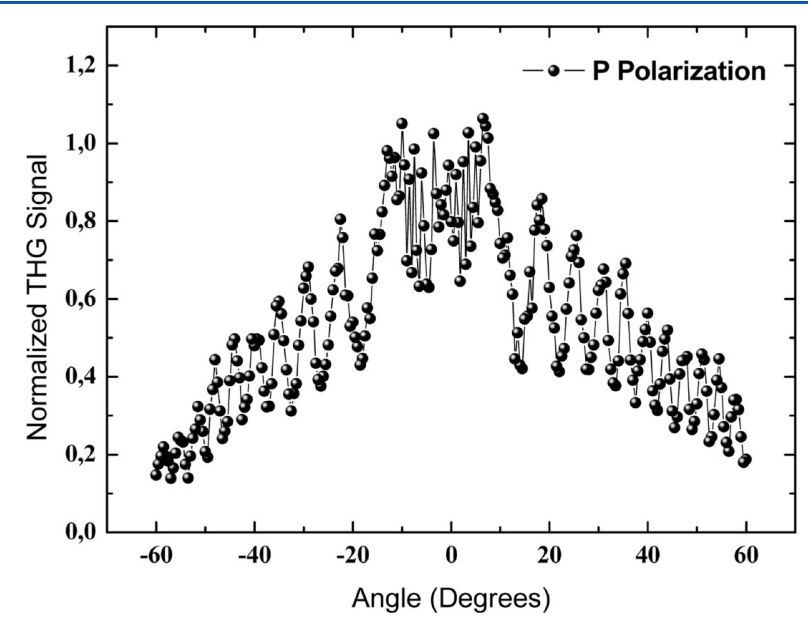

Figure 11. THG Maker fringes curves obtained in the case of $p$ polarization.

of the substrate has been separately measured by in situ removing a part of the film and has been found in all cases to be negligible, compared with the strong nonlinearity emanating from the thin film. Not significant difference has been found between the experimental data obtained for $s$ and $p$ polarizations, so the third-order nonlinear optical susceptibility $\chi^{(3)}$ has been assumed to be the same within the experimental error. The determined effective $\chi^{(3)}$ value was found to be $6.5 \times$ $10^{-20} \mathrm{~m}^{2} \mathrm{~V}^{-2}$.

As it can be seen from all our measurements both the second- and third-order nonlinear optical response of the dye is high which can be attributed to the charge transfer exhibited by this system. The novelty of the chemical structure of the sample renders impossible the comparison with previously published results in the literature. However, it should be mentioned that many studies have been published before concerning the nonlinearity of hemicyanine dyes. ${ }^{21,28,29}$ In these cases big differences have been obtained between the response of the investigated molecular structures, which can be attributed to the different donor/acceptor moieties resulting to enhancement or reduction of the nonlinearity.

\section{CONCLUSIONS}

Successful deposition of high-quality thin films of novel styrylquinolinium dye (E)-1-ethyl-4-(2-(4-hydroxynaphthalen1 -yl)vinyl)quinolinium bromide, having larger conjugated system with respect to styryl dyes possessing benzene and pyridine rings, by means of PLD technique using unconventional high-power $\mathrm{UV} \mathrm{N}_{2}$ laser, at room temperature and low vacuum has been demonstrated, for the first time. The laser energy densities used in this work vary from $250 \mathrm{~mJ} / \mathrm{cm}^{2}$ to 3.5 $\mathrm{J} / \mathrm{cm}^{2}$, and the laser repetition rate was $20 \mathrm{~Hz}$. The films have been found to be homogeneous and without any cracks and droplets on the surfaces, which was verified by the stability of the nonlinear optical response over the surface of the film. The chemical structure of the native material has been preserved in the thin films under the chosen experimental conditions (laser fluence of $250 \mathrm{~mJ} / \mathrm{cm}^{2}$, at $10 \mathrm{~Hz}$ repetition rate), which was verified with FTIR spectroscopic analysis. The pressed microcrystal targets from complex organic NLO dye are found to be suitable for PLD process using vacuum conditions in the range of $10^{-2}-10^{-3}$ mbar. The obtained films are amorphous, and their surface morphology is characterized by small particles and roughness not more than $3 \mathrm{~nm}$ from AFM results, which is still enough and compatible for NLO applications. The second- and third-order nonlinearities of the styrylquinolinium dye thin films were measured by the SHG, Z-scan, and THG techniques, and large nonlinear optical parameters have been found. As a result, the proposed new deposition conditions offer the possibility to prepare thin films with preknown properties, thus tailoring the optical/nonlinear optical properties. This fact combined with the possibility to vary the size of the conjugate system and the type of the N-, 2-, and 4-substituents, further tuning the attribute of the films opens new perspective to utilize the systems in a variety of photonic applications.

\section{AUTHOR INFORMATION}

\section{Corresponding Author}

*E-mail: bouchta.sahraoui@univ-angers.fr; Tel: 02417354 89; Fax: +33241735216.

\section{Notes}

The authors declare no competing financial interest.

\section{ACKNOWLEDGMENTS}

T.K., R.B., and M.T. thank the National Research Fund of Bulgaria (Grant TK DO-02-166) for financial support of this work.

\section{REFERENCES}

(1) Chemla, D. S.; Zyss, J. Nonlinear Optical Properties of Organic Molecules and Crystals; Academic Press: New York, 1987.

(2) Sahraoui, B.; Nguyen Phu, X.; Salle, M.; Gorgues, A. Opt. Lett. 1998, 23, 1811-1813.

(3) Derkowska, B.; Mulatier, J. C.; Fuks, I.; Sahraoui, B.; Nguyen Phu, X.; Andraud, C. J. Opt. Soc. Am. B 2001, 18, 610-616.

(4) Sahraoui, B.; Kityk, I.; Hudhomme, P.; Gorgues, A. J. Phys. Chem. B 2001, 105, 6295-6299. 
(5) El Ouazzani, H.; Iliopoulos, K.; Pranaitis, M.; Krupka, O.; Smokal, V.; Kolendo, A.; Sahraoui, B. J. Phys. Chem. B 2011, 115, 1944-1949.

(6) Ma, Sh.; Lu, X.; Zheng, J.; Wang, W.; Zhang, Z. Thin Solid Films 1995, 254, 263-267.

(7) Marder, S.; Perry, J.; Shaefer, W. Science 1989, 245, 626-628.

(8) Wolff, J.; Wortmann, R. Adv. Phys. Org. Chem. 1999, 32, 1-121.

(9) Nalwa, H. S.; Watanabe, T.; Miyata, S. Organic Materials for Second-Order Nonlinear Optics. In Nonlinear Optics of Organic Molecules and Polymers; Nalwa, H. S., Miyata, S., Eds.; CRC Press: Boca Raton, FL, 1997; Chapter 4, pp 89-351.

(10) Sanches, C.; Lebean, B.; Chaput, F.; Boilot, J. P. Adv. Mater. 2003, 15, 1969-1994.

(11) Quintel, A.; Budde, F.; Rechsteiner, P.; Thoma, K.; Zayatsb., A.; Hulliger, J. J. Mater. Chem. 2000, 10, 27-30.

(12) Chrisey, D. B.; Pique, A.; McGill, R. A.; Horwiz, J. S.; Ringeisen, B. R.; Bubb, D. B.; Wu, P. K. Chem. Rev. 2003, 103, 553-576.

(13) Sheik-Bahae, M.; Said, A. A.; Wie, T.-H.; Hagan, D. J.; Van Stryland, E. W. IEEE J. Quantum Electron. 1990, 26, 760-769.

(14) Herman, W. N.; Hayden, L. M. J. Opt. Soc. Am. B 1995, 12, 416-427.

(15) Jerphagnon, J.; Kurtz, S. K. J. Appl. Phys. 1970, 41, 1667-1681.

(16) Sahraoui, B.; Luc, J.; Meghea, A.; Czaplicki, R.; Fillaut, J.-L.; Migalska-Zalas, A. J. Opt. A: Pure Appl. Opt. 2009, 11, 1-26.

(17) Serbezov, V.; Benacka, St; Hadgiev, D.; Atanasov, P.; Electronov, N.; Smatko, V.; Stribik, V.; Vassilev, N. J. Appl. Phys. 1990, 67, 6953-6957.

(18) Mortazavi, M. A.; Knoesen, A.; Kowel, S. T.; Higgins, B. G.; Dienes, A. J. Opt. Soc. Am. B 1989, 6, 733-741.

(19) Singer, K. D.; Sohn, J. E.; Lalama, S. J. Appl. Phys. Lett. 1986, 49, 248-250.

(20) Kubodera, K.; Kobayashi, H. Mol. Cryst. Liq. Cryst. 1990, 182, $103-113$.

(21) Qin, C.; Wang, X.; Wang, J.-J.; Mao, J.; Yang, J.; Dai, L.; Chen, G. Dyes Pigm. 2009, 82, 329-335.

(22) Fromherz, P. J. Phys. Chem. 1995, 99, 7188-7192.

(23) Binnemans, K.; Bex, C.; Venard, A.; De Leebeeck, H.; GörllerWalrand, C. J. Mol. Liq. 1999, 83, 283-294.

(24) Bruni, S.; Cariati, E.; Cariati, F.; Porta, F. A.; Quici, S.; Roberto, D. Spectrochim. Acta, Part A 2001, 57, 1417-1426.

(25) Jedrzejewska, B.; Kabatc, J.; Pietrzak, M.; Paczkowski, J. Dyes Pigm. 2003, 58, 47-58.

(26) Reichardt, C. Solvents and Solvent Effects in Organic Chemistry, Third, Updated and Enlarged ed.; VCH: New York, 2003.

(27) Horcas, I.; Fernandez, R.; Gomez-Rodriguez, J. M.; Colchero, J.; Gomez-Herrero, J.; Baro, M. Rev. Sci. Instrum. 2007, 78, 013705.

(28) Marowsky, G.; Chi, L. F.; Möbius, D.; Steinhoff, R.; Shen, Y. R.; Dorsch, D.; Rieger, B. Chem. Phys. Lett. 1988, 147, 420-424.

(29) Schildkraut, J. S.; Penner, T. L.; Willand, C. S.; Ulman, A. Opt. Lett. 1988, 13, 134-136. 